Dinkar Sharma*, Ramandeep Kaur, and Honey Sharma

\title{
Investigation of thermo-elastic characteristics in functionally graded rotating disk using finite element method
}

https://doi.org/10.1515/nleng-2021-0025

Received Mar 30, 2021; accepted Aug 3, 2021.

Abstract: In this research paper, displacement, stresses and strains are presented for rotating FGM disk with variable thickness by using finite element method (FEM). Thermo-elastic material properties and thickness of FGM disk continuously vary as exponential and power law function in radial direction along radius of disk. The value of Poisson's ratio is taken as constant. The problem of thermo-elasticity is converted into second order governing differential equation in terms of radial coordinate. This conversion is based upon equilibrium equation for disk and stress-strain relationship. The influence of variable thickness, angular velocity and functionally graded materials is discussed on thermo-elastic characteristics of rotating disk for exponential variation of material properties. Further, these thermo-elastic characteristics of disk are plotted for various values of non-homogeneity parameter under power law distribution of material properties. Thus, the investigations done in this research paper may be useful for industrial area in construction an appropriate FGM disk by controlling above mentioned parameters.

Keywords: FGM, FEM, thickness, stress, strain and material properties

\section{Introduction}

Functionally graded materials are a new class of composites that are also known as multifunctional materials due to their spatial variation composition. The positive impact of compressive residual surface stresses on wear

\footnotetext{
*Corresponding Author: Dinkar Sharma, Department of Mathematics, Lyallpur Khalsa College, Jalandhar, India, E-mail: dinkar.nitj@gmail.com

Ramandeep Kaur, Department of Mathematics, I.K.G. Punjab Technical University, Kapurthala, India

Honey Sharma, Department of Mathematics, Gulzar group of Institute, Khanna, India
}

resistance as well as on strength may be counted as a potential advantage of these multifunctional materials. So, it become a matter of concern to design an appropriate blueprint of the progressive compositional gradient for an optimal distribution of the residual stresses to correlate with mechanical properties [1]. Composite materials have found vast applications in distinguished fields of structural engineering such as aerospace, automobile, bio-medical, civil structures, etc. [2]. In the past few decades, many researchers have investigated the behavior of these multifunctional materials in the presence of different environmental conditions. Wang and $\mathrm{Zu}[3,4]$ published and concluded that the damping, translational speed, and the excitation amplitude significantly affect the nonlinear dynamical responses of the translational FG plate using an analytical and numerical method approach. The nonlinear vibration analysis of rotating functionally graded cylindrical shell has been reported by Sheng and Wang [5]. Liew et al. [6] have studied the nonlinear vibration behaviour of a laminated FG cylindrical panel. Talha and Singh [7] have presented the nonlinear free flexural vibration analysis of an FG plate using an improved higher-order theory. Akbari and Ghanbari [8] studied influence of internal pressure, thermal load and rotation on functionally graded hollow discs from exact analytical solution under radially varying material properties. Kalali et al. [9] derived elasto-plastic stress solution numerically in axisymmetric functionally graded rotating disk, cylindrical and spherical vessel with help of Hencky's stress-strain relation. Salehi et al. [10] used linear plane elasticity theory to find analytical solution for axisymmetric thick-walled FGM cylinders. Habib et al. [11] made stress and strain analysis in functionally graded cylinder under exponentially varying material properties by finite element method and ANASYS software. Yadav and Jiwari $[12,13]$ used finite element to solve differential equations in various mathematical models. Afsar and Go [14] shown effect of radial thickness, angular speed and temperature profile on thermoelastic field in a thin circular FGM disk for exponentially varying material properties. Khorsand and Tang [15] used differential quadrature method and co-evolutionary particle 
swarm optimization approach to minimize stress and displacement in functionally graded hollow circular disk under variable thickness. Go [16] derived second order differential equation based upon two-dimensional thermal elastic theory to study stress, strain and temperature distribution in rotating circular disk. Thawati et al. [17] represented stress and deformation in functionally graded disk for three types of material properties named as: mori-tanaka scheme, power law distribution and exponential distribution under variable thickness. Zheng et al. [18] developed stress field numerically by using finite difference method in functionally graded rotating disk. To carried out stress field material properties are assumed to followed power law distribution. Nejad et al. [19] obtained elasto-plastic deformation and stress from exact form of analytical solution in FGM rotating disk for constant Poisson's ration. Calliogue et al. [20] made elastic-plastic stress analysis in functionally graded rotating disk from analytical and numerical solution for different values of angular velocities under radially varying material properties. Kordkheili and Livani [21] used material properties as function of temperature to study thermoelastic creep behaviour in functionally graded rotating disk with variable thickness. Arnab et al. [22] employed power and exponential function variation in radial direction to study thermoelastic field in thin circular FGM disk. Allam et al. [23] used infinitesimal theory to drive accurate and effective solutions for displacement and stress in rotating annular disk with variable thickness. Jalali and Shahriari [24] obtained stresses and deformation in rotating variable disk for three types of boundary conditions by finite difference method. Torabnia et al. [25] shown effect of young's modulus, density and yield stress on stress, strain and radial displacement in FGM hollow rotors from analytical solution of equilibrium equation. Garg [26] carried out elastic stress and strain in rotating FGM disk for different values of thickness gradation index under variable material properties in radial direction. Abdalla et al. [27] studied thermomechanical stress in functionally graded rotating disk with finite element method in two-dimensional model. Kursun et al. [28] made stress analysis in FGM discs under condition of uniform pressure on inner surface and linearly decreasing temperature distribution for radially varying material properties. Nkene et al. [29] considered second law of Newton, Hooke's law and stress-strain relationship to drive analytical and numerical solution in FGM rotating hollow cylinder for different values of inhomogeneity parameter. Saadtfar [30] analysed effect of angular velocity, hygrothermal loading and moisture concentration on piezomagnetic rotating thick walled cylinder for hygrothermal boundary conditions. Abrinia et al. [31] assumed material properties varying along thickness as power law function to obtained radial and circumferential stress in FGM cylinder under internal pressure and temperature. Rahimi and Nejad [32] investigated thermal stresses from an exact solution in FGM rotating hollow thick-walled cylinder under internal and external pressure. Evci and Gulgec [33] derived analytical solution to represent stresses and displacement in FGM hollow cylinder by using airy stress function. Further, failure analysis done with coulomb-mohr theory and tresca yield criterion. Mantena et al. [34] obtained solution in form of bessels and trigonometric functions to study heat conduction and thermal stresses in hollow cylinder under non-homogeneous material properties. Yildirim [35] presented thermomechanical analysis analytically with Navier equations under assumption of spherically-symmetric plain strain for non-homogeneous isotropic spheres. Farhan et al. [36] proposed finite difference method to study thermoelasticity in isotropic and infinitely long circular cylinder under temperature dependent material properties. Hu et al. [37] investigated influence of various parameters on natural vibration in rotating functionally graded ring plate. Go [38] made thermoelastic characteristics analysis numerically under variable contact force and homogeneous thickness. Thakare and Warbhe [39] studied temperature distribution and stress distribution in thick hollow cylinder for homogeneous and inhomogeneous material properties. Saeedi et al. [40] used successive approximation method to study stresses and strains in thick walled FGM cylindrical shells under internal pressure and temperature gradient. In the present work, rotating disks of functionally graded materials are constructed due to its utilization in different fields of Engineering and Science. A stress field in FGM isotropic and anistropic cylinder under internal pressure and uniform heat generation for different values of gradient index, is represented. These disks are modelled under variety of thermo-mechanical properties to extend our published work [41-45].

Basic Equations of the problem: For rotating disk, the strain-displacement relationship irrespective of thickness can be written as:

$$
\varepsilon_{r}=\frac{d u}{d r}, \varepsilon_{\theta}=\frac{u}{r}
$$

Where $u$ is displacement, $r$ is radial coordinate and $\left(\varepsilon_{r}, \varepsilon_{\theta}\right)$ are strains in radial and circumferential direction.

For elastic deformation, by using Hooke's law relations between strains and stresses can be written as:

$$
\sigma_{r}=\frac{E(r)}{1-v^{2}}\left[\varepsilon_{r}+v \varepsilon_{\theta}\right]
$$




$$
\sigma_{\theta}=\frac{E(r)}{1-v^{2}}\left[\varepsilon_{\theta}+v \varepsilon_{r}\right]
$$

where $\left(\sigma_{r}, \sigma_{\theta}\right)$ represents stresses in radial and circumferential direction, $E(r)$ is young's modulus and $v$ is Poisson's ration of disk.

Equilibrium equation for disk of variable thickness is given as:

$$
\frac{d}{d r}\left[h(r) r \sigma_{r}\right]-h(r) \sigma_{\theta}+h(r) \rho(r) \omega^{2} r^{2}=0
$$

where $\omega$ is angular velocity, $\rho(r)$ is density of rotating disk and $h(r)$ is thickness of disk.

\section{Case I: Exponential variation of material properties}

In this section, we assumed that material properties of rotating disk are varying exponentially along radial direction. Therefore, $E(r), \rho(r)$ and $h(r)$ vary as:

$$
\begin{aligned}
& E(r)=E_{0} e^{-m(r-a) / b} \\
& \rho(r)=\rho_{0} e^{-n(r-a) / b} \\
& h(r)=h_{0} e^{-k(r-a) / b}
\end{aligned}
$$

Where $\mathrm{r}$ is radial coordinate, $E_{0}, \rho_{0}$ and $h_{0}$ are constants of young's modulus, mass density and thickness respectively at inner surface of FGM disk. The index $m$ and $n$ are material parameters, the $k$ is the geometric parameter.

After substituting values from (5-7) into equation (4), governing differential equation of problem in terms of radial displacement become:

$$
r^{2} \frac{d^{2} u}{d r^{2}}+r(1-r P) \frac{d u}{d r}-(1+r v P) u=Q r^{3} e^{-R(r-a)}
$$

Where $P=\frac{k+m}{b}, Q=\frac{\rho_{0}}{e_{0}}\left(1-v^{2}\right) \omega^{2}$ and $R=\frac{-(n-m)}{b}$

\section{Case II: Power law variation of material properties}

In this case, to study thermoelastic characteristics in FGM disk material properties of disk are consider to follow power law variation along radius as follows:

$$
\begin{aligned}
& E(r)=E_{0} r^{n_{1}} \\
& \rho(r)=\rho_{0} r^{n_{2}} \\
& h(r)=h_{0} r^{n_{3}}
\end{aligned}
$$

By substituting values of various material parameters from Eqs. (9-11) into Eq. (4), the differential equation which represents problem of thermoelasticity is obtained, as:

$$
\begin{aligned}
& \frac{d^{2} u}{d r^{2}}+\frac{n_{1}+n_{3}+1}{r} \frac{d u}{d r}+\frac{1}{r^{2}}\left(v\left(n_{1}+n_{3}\right)-1\right) u \\
& +\frac{\rho_{0}}{E_{0}}\left(1-v^{2}\right) \omega^{2} r^{n_{2}-n+1}=0
\end{aligned}
$$

After simplification equation (15), we get:

$$
\frac{d^{2} u}{d r^{2}}+\frac{P}{r} \frac{d u}{d r}+\frac{Q}{r^{2}} u+R r^{n_{2}-n+1}=0
$$

Where $P=n_{1}+n_{3}+1, Q=v\left(n_{1}+n_{3}\right)-1$ and $R=\frac{\rho_{0}}{E_{0}}\left(1-v^{2}\right) \omega^{2}$

\section{Finite element formulation}

In this section, FEM is applied to Eq. (8) and (13). To solve these equations standard discretization approach of finite element method is used. After applying FEM differential equation is converted into simultaneous linear equation as:

$$
\sum_{j=1}^{2} K_{i j}{ }^{e} F_{j}{ }^{e}=L_{i}{ }^{e} ; i=1,2 ; e=1,2,3, \ldots, n
$$

\section{Case I:}

$$
\begin{aligned}
& K_{i j}^{e}=\left[\phi_{i} \frac{d \phi_{j}}{d r}\right]_{r_{e}}^{r_{e+1}}-\int_{r_{e}}^{r_{e+1}} \frac{d \phi_{i}}{d r} \frac{d \phi_{j}}{d r} d r+\int_{r_{e}}^{r_{e+1}} \phi_{i}\left(\frac{1}{r}-P\right) \frac{d \phi_{j}}{d r} d r \\
& +\int_{r_{e}}^{r_{e+1}} \phi_{i} \phi_{j}\left(\frac{v P}{r}+\frac{1}{r^{2}}\right) d r
\end{aligned}
$$

$$
L_{i}^{e}=Q \int_{r_{e}}^{r_{e+1}} \phi_{i} r e^{-R(r-a)} d r
$$

Case II:

$K_{i j}^{e}=\left[\phi_{i} \frac{d \phi_{j}}{d r}\right]_{r_{e}}^{r_{e+1}}-\int_{r_{e}}^{r_{e+1}} \frac{d \phi_{i}}{d r} \frac{d \phi_{j}}{d r} d r+\int_{r_{e}}^{r_{e+1}} \frac{\phi_{i}}{r} \frac{d \phi_{j}}{d r} d r+\int_{r_{e}}^{r_{e+1}} \frac{\phi_{i} \phi_{j}}{r^{2}} d r$

$$
L_{i}^{e}=R \int_{r_{e}}^{r_{e+1}} \phi_{i} r^{n_{2}-n_{1}+1} d r
$$

where $\varphi_{1}^{e}=\frac{r_{e+1}-r}{r_{e+1}-r_{e}}, \varphi_{2}^{e}=\frac{r-r_{e}}{r_{e+1}-r_{e}}$ 


\section{Numerical results and discussions}

In this section, numerical results for thermo-elastic characteristics have been presented graphically for rotating FGM disk under exponential and power-law distribution of material properties. The value of Poisson's ratio is taken as 0.3 constant throughout material.

\section{Case I:}

In this section, an effect of angular velocity, thickness and functionally graded materials study on stresses, strains and displacement under exponentially varying material properties.

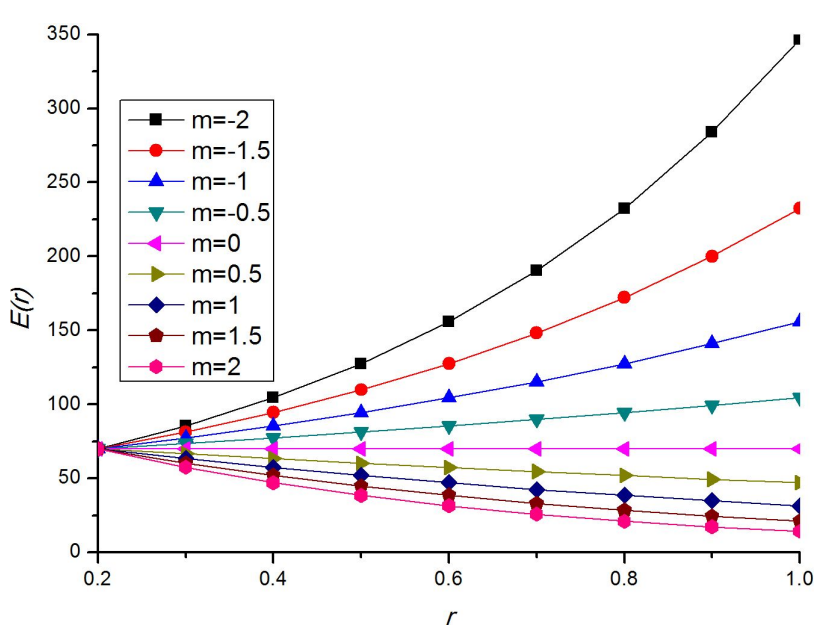

Figure 1: Young's modulus versus radial coordinate

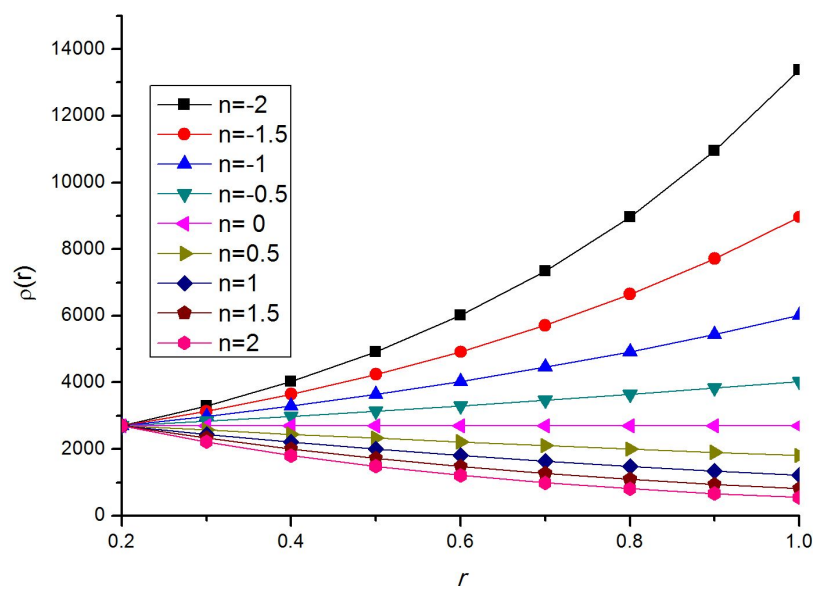

Figure 2: Density versus radial coordinate

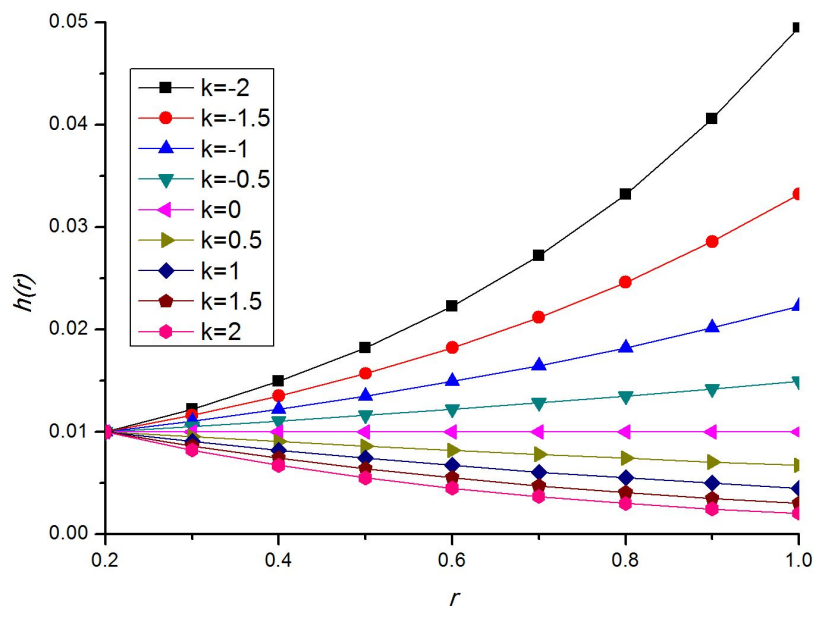

Figure 3: Thickness versus radial coordinate

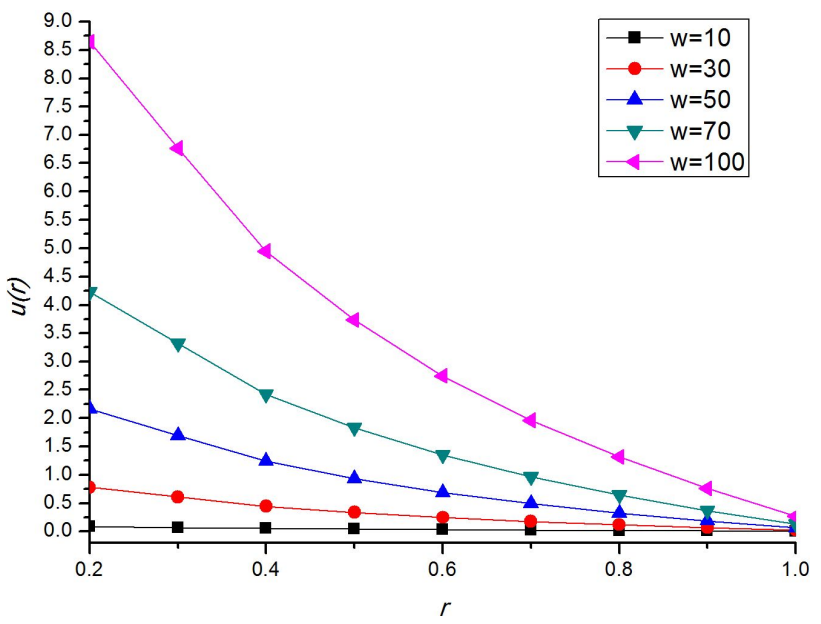

Figure 4: Displacement against radial coordinate for different values of angular velocity

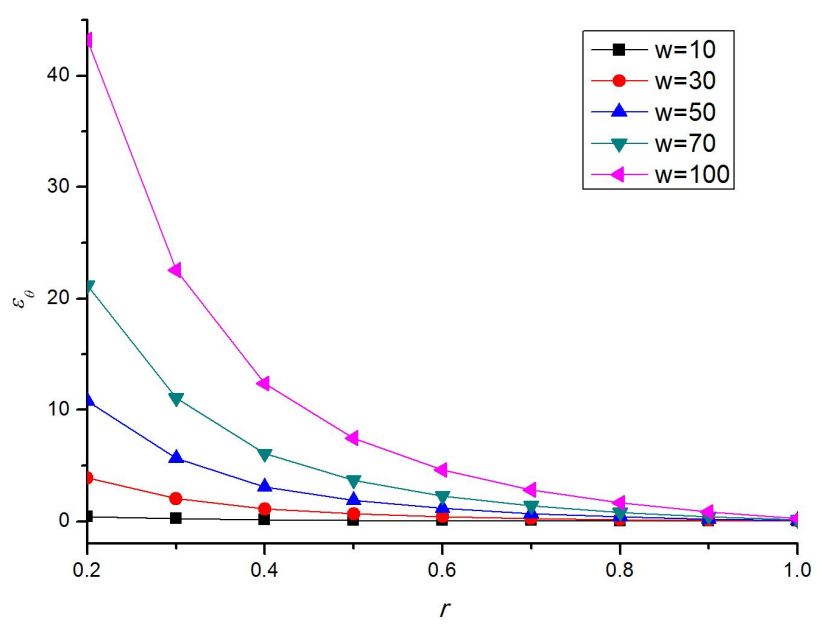

Figure 5: Circumferential strain against radial coordinate for different values of angular velocity 


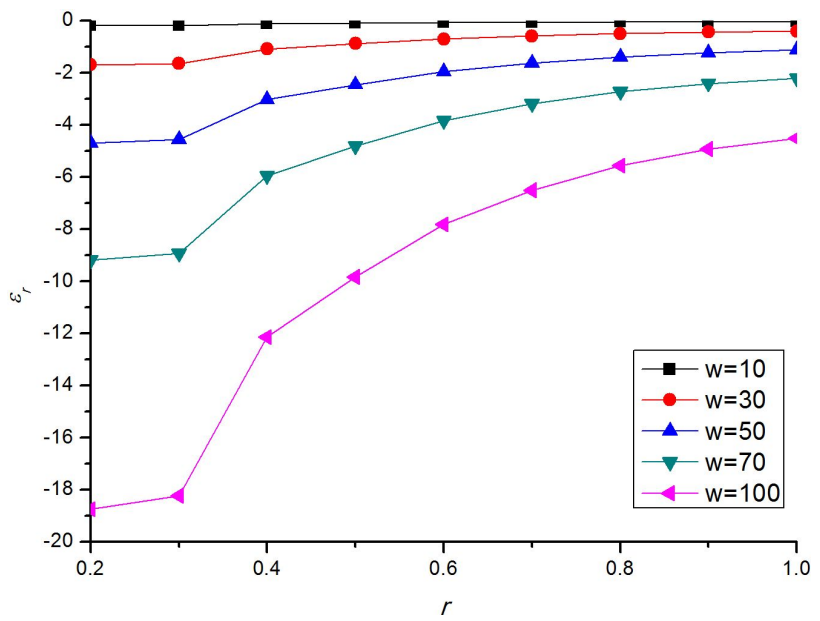

Figure 6: Radial strain against radial coordinate for different values of angular

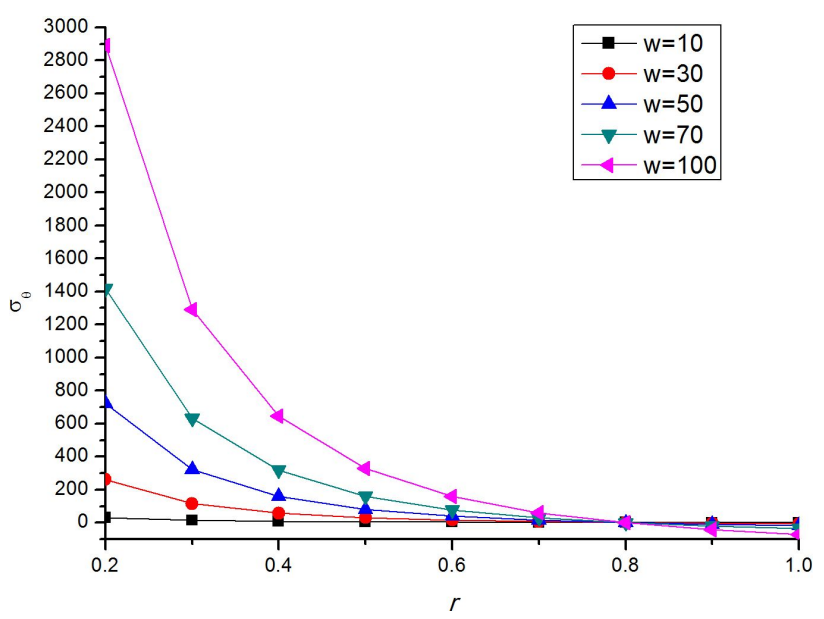

Figure 7: Circumferential stress against radial coordinate for different values of angular velocity

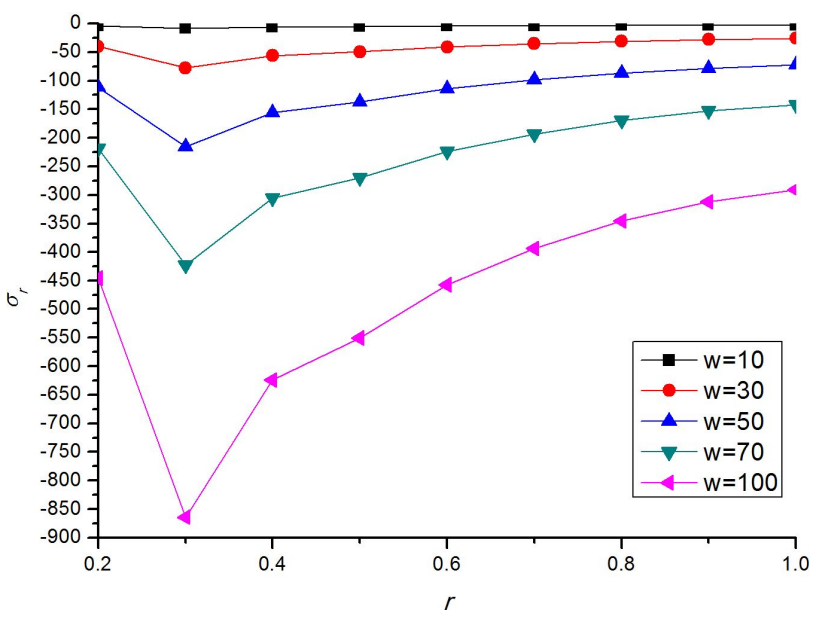

Figure 8: Radial stress against radial coordinate for different values of angular

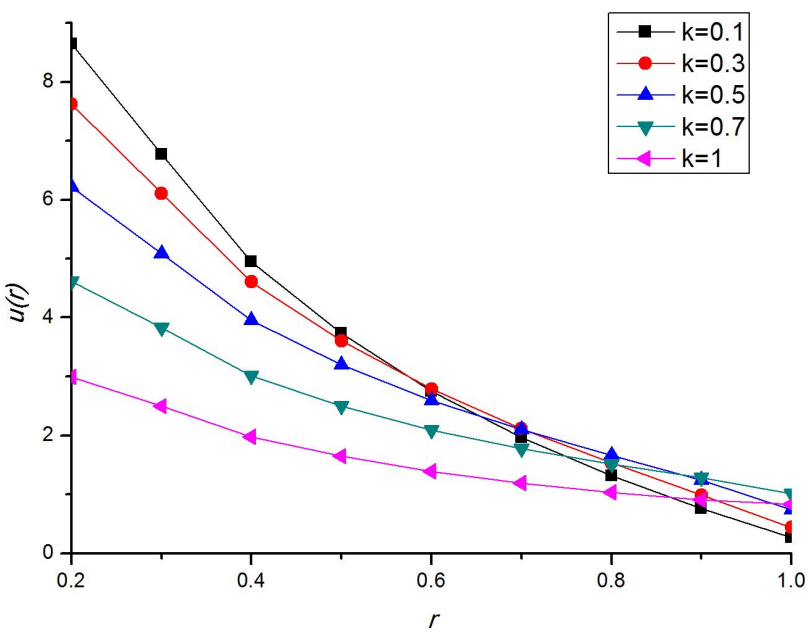

Figure 9: Displacement versus radial coordinate for variable thickness

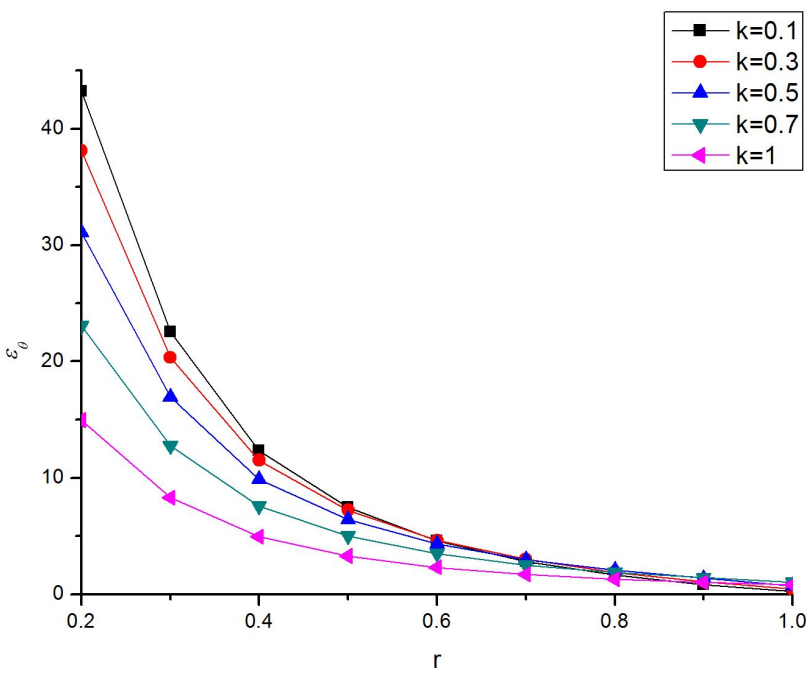

Figure 10: Circumferential strain versus radial coordinate for variable thickness

Figures $1-3$ present variation in thermomechanical material properties along radial direction in rotating FGM disk. From Figure 1, we can see that as value of $m$ increases, modulus of elasticity decreases. Maximum variation in $E(r)$ exists when $m=-2$. From Figure 2 and 3, one can observe that behaviour of density and thickness curves are same in nature. The maximum value of density and thickness is obtained for minimum value of parameter. For +ve values of parameter behaviour of all material properties are decreasing in nature but for -ve values of parameter behaviour is increasing in nature.

Figures $4-8$ summarize variation in thermoelastic characteristics for various values of angular velocity against radial coordinate $r$. Figure 4 is evident displacement for different values of angular velocity when $0.2 \leq$ $r \leq 1$. It is clear that as value of angular velocity increases, 


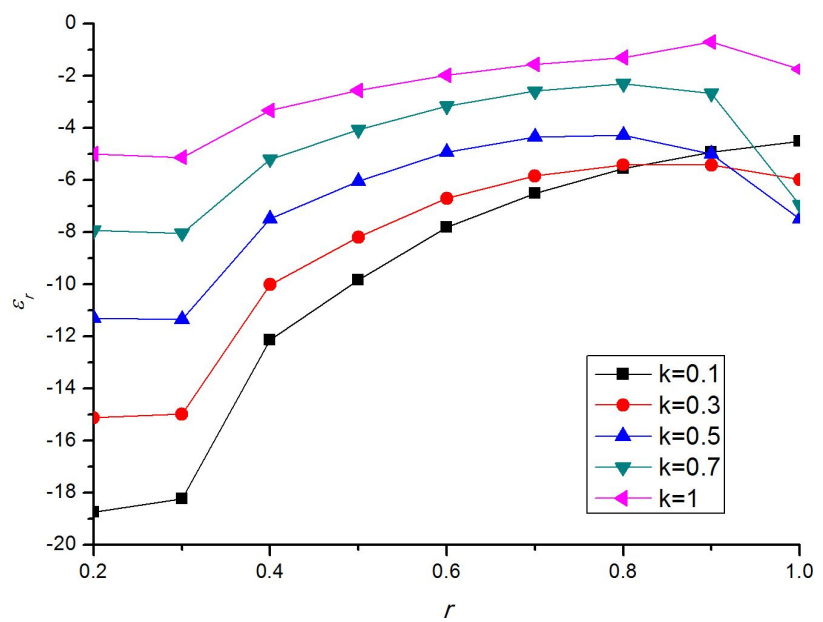

Figure 11: Radial strain versus radial coordinate for variable thickness

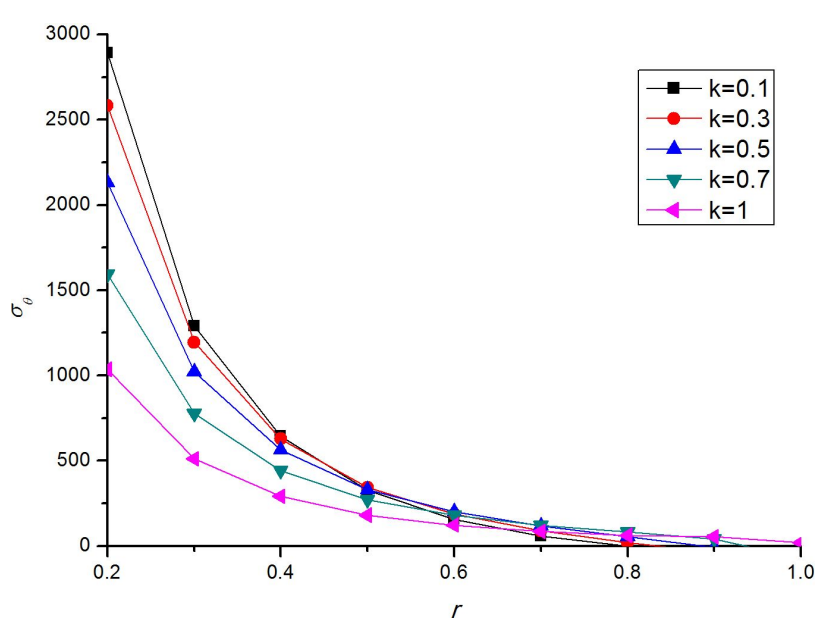

Figure 12: Circumferential stress versus radial coordinate for variable thickness

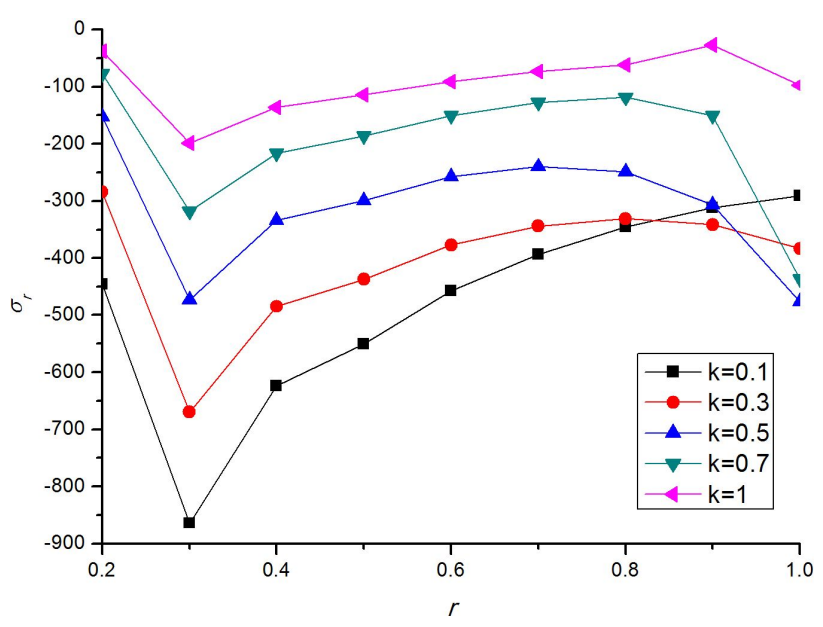

Figure 13: Radial stress versus radial coordinate for variable thickness

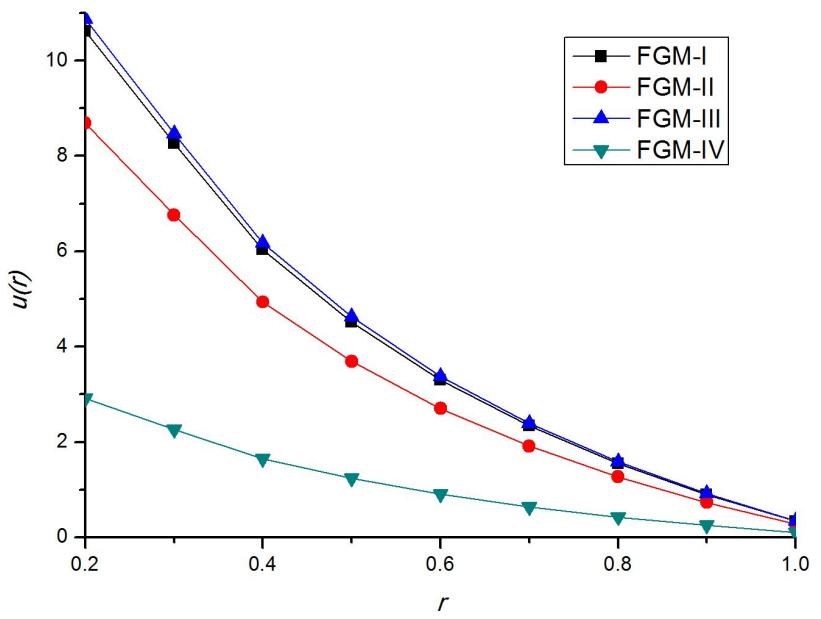

Figure 14: Displacement for radial coordinate for different FGM's

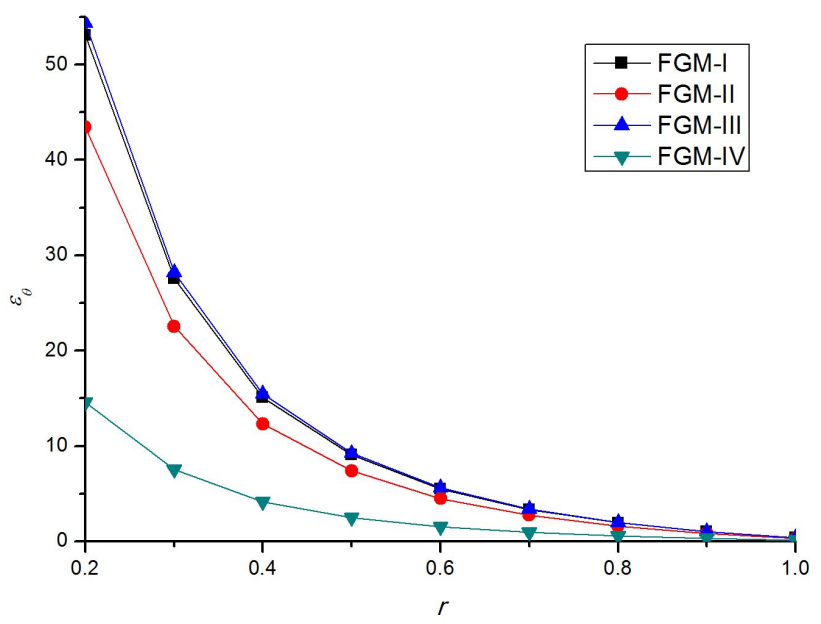

Figure 15: Circumferential strain radial coordinate for different FGM's

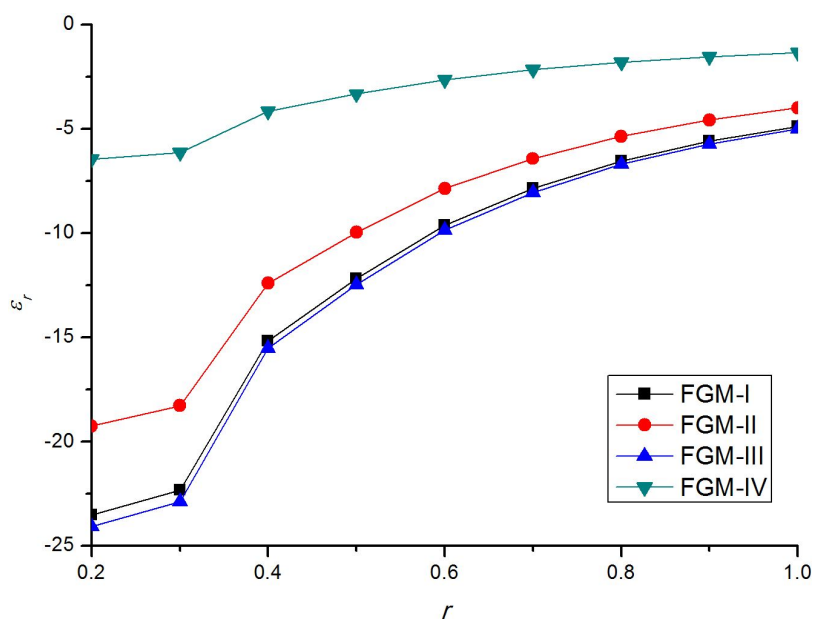

Figure 16: Radial strain radial coordinate for different FGM's 


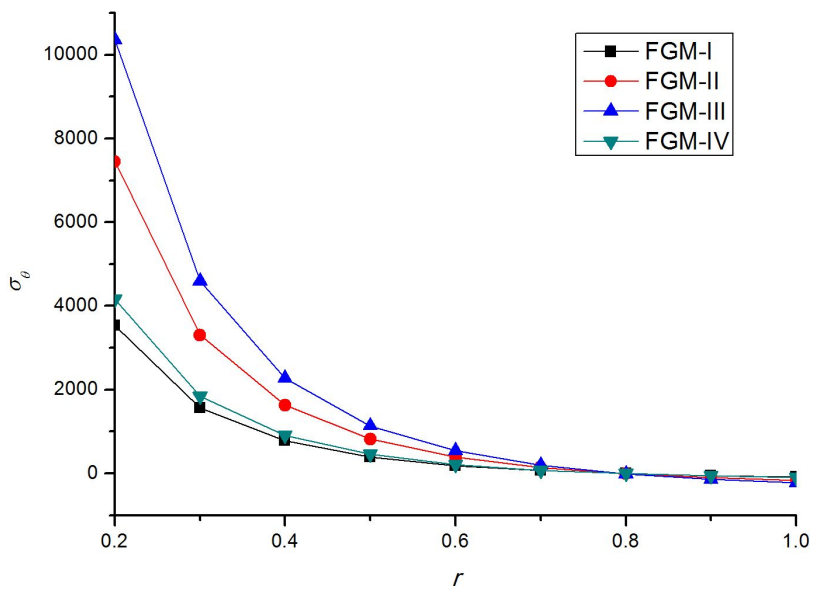

Figure 17: Circumferential stress radial coordinate for different FGM's

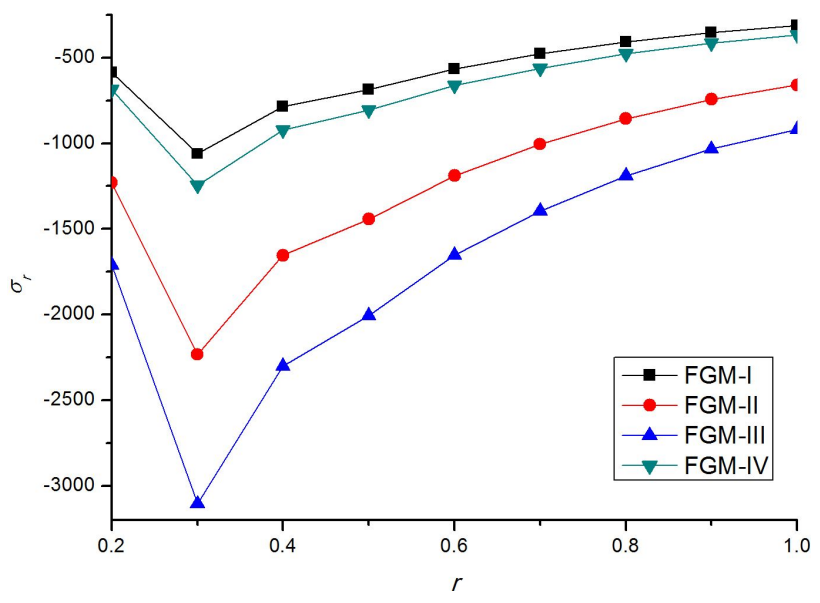

Figure 18: Radial stress radial coordinate for different FGM's

the displacement also increases. For various values of $\omega$ maximum displacement exists for minimum value of $r$. Figure 5 reveals that the behaviour of circumferential strain and displacement curves are same for different values of $\omega$. The maximum and minimum numerical value of circumferential strain exists corresponding maximum and minimum value of angular velocity respectively. The displacement and circumferential strain curves are tensile for inner surface of disk. Figure 6 exhibits that, radial strain and angular velocity are opposite in nature for different values of $r$ i.e $\varepsilon_{r}$ decreases as value of $r$ increases. Figure 7, illustrate circumferential stress against radial coordinate for different values of $\omega$. The behaviour of circumferential stress curves are changes at $r=0.8$. At inner surface of disk maximum value of circumferential stress is obtained for maximum value of angular velocity but at outer surface of disk maximum value is achieved for minimum value for angular velocity. Figure 8 represents, effect of angular ve- locity on radial stress. For $\omega=100$ radial stress curve is very fluctuating in nature but for $\omega=10$ curve shows steady behaviour.

Figures $9-13$ show the effect of thickness on thermoelastic characteristics by taking different values of thickness parameter $k$. As per Figure 9, for inner surface of disk maximum value of displacement is obtained corresponding to $k=0.1$ and for outer surface maximum value it exists for $k=0.7$. Figure 10 shows that, circumferential strain curves are more variable for inner surface of disk. All circumferential curves are decreasing in nature i.e. as $r$ increases $\varepsilon_{\theta}$ decreases. But from Figure 11, one can see that behaviour of radial strain curve is increasing and maximum value of radial strain is achieved corresponding to maximum value of thickness parameter. From Figures 12 and 13 it is noted that behaviour of circumferential and radial stress curves are different in nature. Maximum value of circumferential stress and minimum value of radial stress are obtained for $k=0.1$. Also, circumferential stress is maximum at inner surface of disk but radial stress is minimum at this surface.

Figure $14-18$ show distribution of thermo-elastic characteristics of rotating disk that disk is constructed from different FGM's. The thermo-mechanical properties of FGM's are presented in Table 1.

From Figure 14, it is observed that displacement decreases as value of radial coordinate increases. The maximum value of displacement is obtained for stainless steel and minimum value is attained for silicon nitride. Figure 15 displays that circumferential strain converges to zero as radial coordinate moves toward outer surface of disk. Radial strain curves are increasing in nature, according to Figure 16. The behaviour of all radial strain curves are linear when $0.2 \leq r \leq 0.3$ and for remaining region behaviour is non-linear in nature. The circumferential stress is maximum for silicon nitride and least for aluminimum. The behaviour of displacement and circumferential strain curves are same in nature. As we can see from Figure 18, radial stress curve is firstly linearly decreasing in nature for $0.2 \leq r \leq 0.3$ and it is decreasing for remaining values of radial coordinate.

\section{Case II:}

In this section, graphical representation of thermoelastic characteristics of rotating disk is done under exponential material properties. The effect of non-homogeneity index $\left(n_{1}=n_{2}=n_{3}=n\right)$ is shown on stress, strain and displacement by taking its various values.

Figures 19 - 26 show thermo-mechanical properties and thermo-elastic characteristics for power law distribution of material properties. The effect of non-homogeneity 
Table 1: Thermo-mechanical properties of various functionally graded materials

\begin{tabular}{l|l|l|l}
\hline $\begin{array}{l}\text { Material abbrevia- } \\
\text { tion }\end{array}$ & Material & $E_{0}(\mathrm{GPa})$ & $\rho_{0}\left(\mathrm{~g} / \mathrm{cm}^{3}\right)$ \\
\hline FGM-I & Aluminimum & 71 & 2.70 \\
FGM-II & Zirconium oxide & 180 & 5.68 \\
FGM-III & Stainless steel & 200 & 7.9 \\
FGM-IV & Silicon nitride & 300 & 3.17 \\
\hline
\end{tabular}

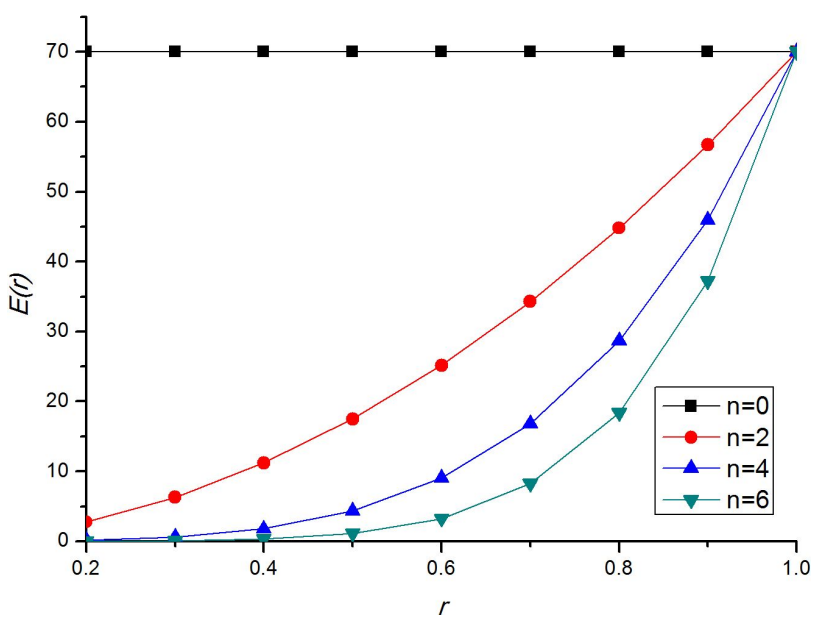

Figure 19: Distribution of Young's modulus with radial coordinate

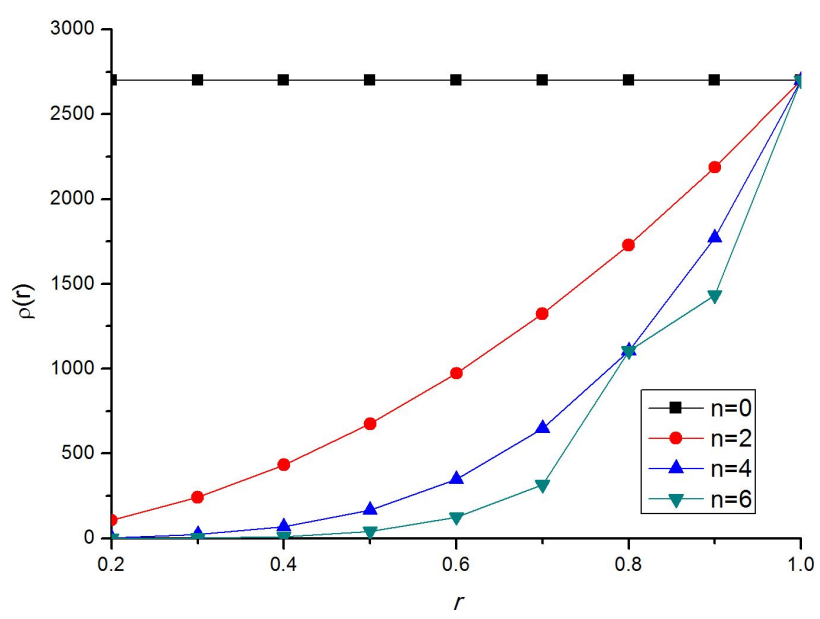

Figure 20: Distribution of density with radial coordinate

parameter is shown by taking different values of $n$. From Figures $19-21$, one can see that maximum value of material properties obtained for $n=2$ and minimum values are obtained for $n=6$. Modulus of elasticity, density and thickness are constant when non homogeneity parameter is absent from material properties. As Figure 22 shows, displacement curve is decreasing for $n=4$ and $n=6$ but for $n=2$ curve is strictly increasing. Maximum displacement is obtained when material properties follow quadratic variation along radius of disk. For $n=2$ circum-

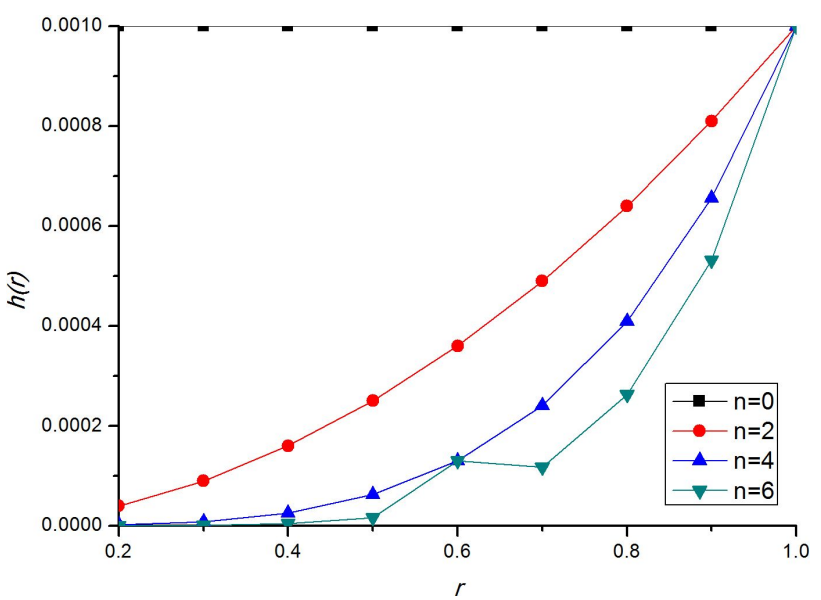

Figure 21: Distribution of thickness with radial coordinate

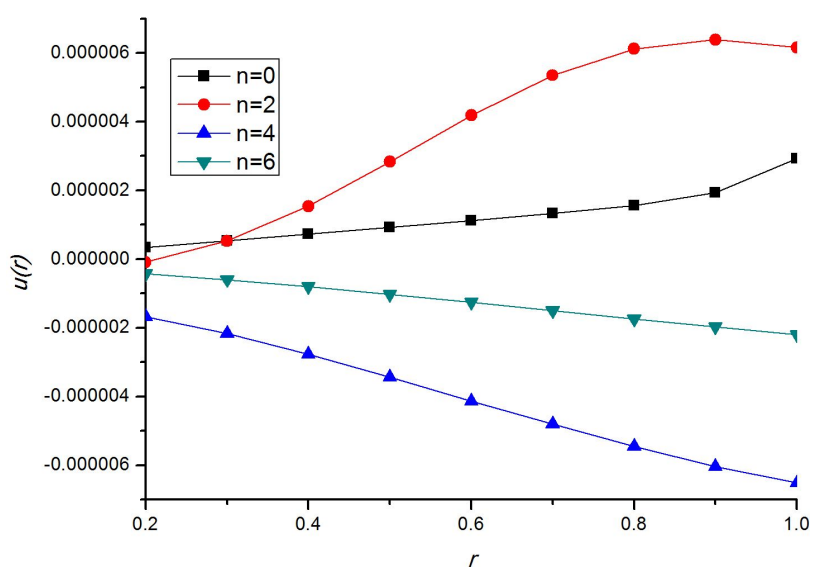

Figure 22: Distribution of displacement with radial coordinate

ferential strain Curve shows increasing behaviour in nature for inner surface of rotating disk but decreasing for outer surface of disk. For $n=0, n=4$ and $n=6$ behaviour of circumferential strain linear and stable. Radial strain is fluctuating for $n=0$ and $n=2$ but this behaviour is linear for $n=6$ and very less elastic for $n=4$. From Figures $25-26$, it is noted that both stress curves are increasing in nature for $n=0$ but shows decreasing behaviour for remaining values of $n$. Maximum variation in stresses exits for outer surface of disk. 


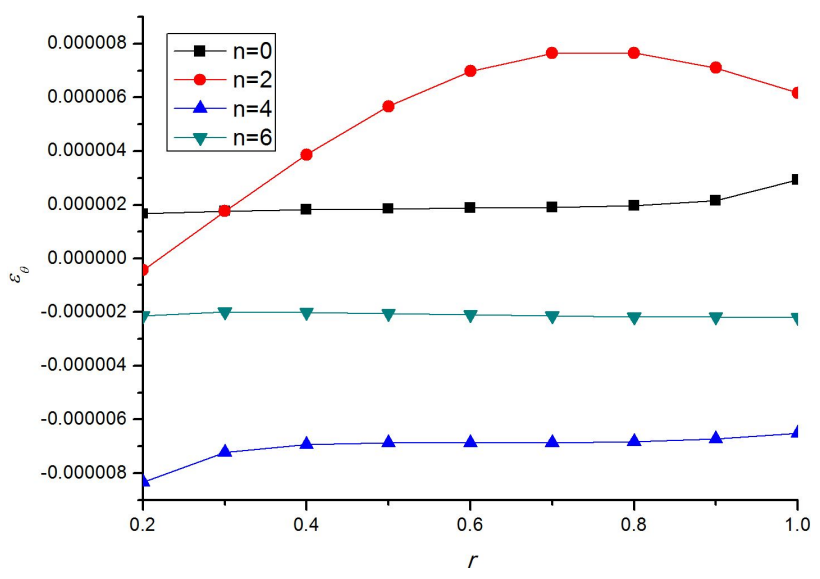

Figure 23: Distribution of circumferential strain with radial coordinate

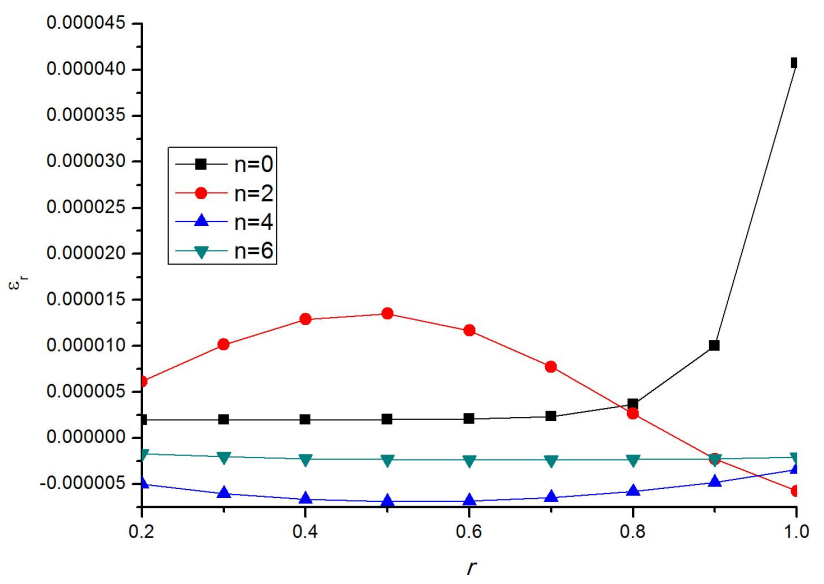

Figure 24: Distribution of radial strain with radial coordinate

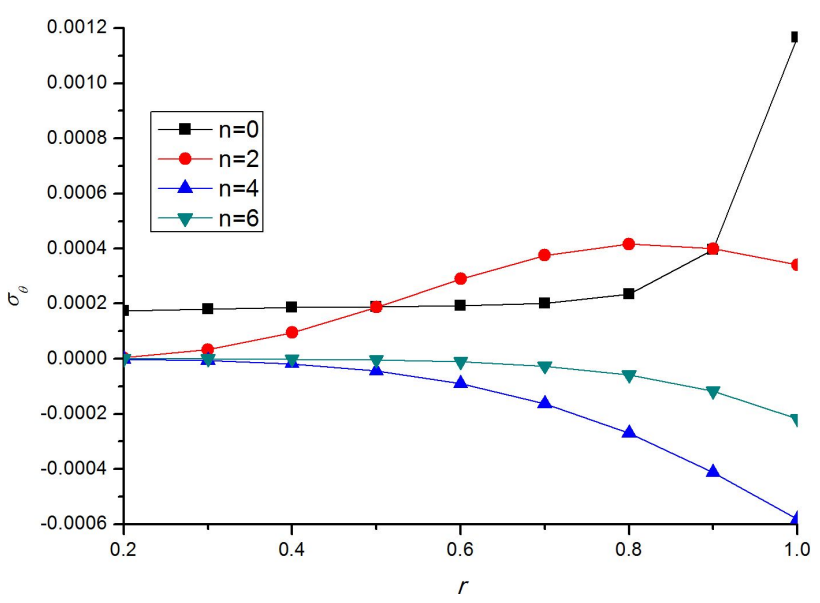

Figure 25: Distribution of circumferential stress with radial coordinate

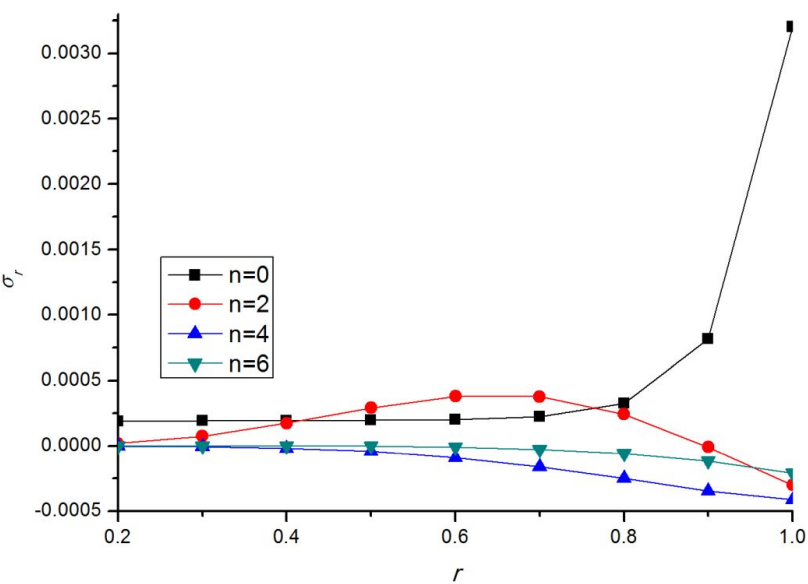

Figure 26: Distribution of radial stress with radial coordinate

\section{Conclusion}

In presented research, thermo-elastic characteristics analysis is made for different material properties profiles in rotating disk which is constructed from FGM material. Finite element method is employed to find displacement, strains and stresses numerically from equilibrium equation. The following conclusion can be made:

1) In exponential distribution of material properties, the value of thermo-mechanical properties increases as material parameter increases but for power law distribution this behaviour of material properties is opposite in nature.

2) Maximum variation in displacement, strains and stresses exist for inner surface of rotating disk when thickness is taken as variable parameter in case-I.

3) The behaviour of thermo-elastic characteristics is same in nature but differ numerically for different types of functionally graded materials.

4) In power law distribution of material properties maximum value of thermo-elastic characteristics is obtained for $n=2$.

5) For $n=4$ and $n=6$ thermo-elastic curves are more stable in nature in power law distribution of material properties.

Finally, these findings may be helpful in construction/designing of FGM rotating disks, which have a numerous application in different fields of engineering.

Funding information: The authors state no funding involved. 
Author contributions: All authors have accepted responsibility for the entire content of this manuscript and approved its submission.

Conflict of interest: The authors state no conflict of interest.

\section{References}

[1] Low IM. Advances in ceramic matrix composites, 2nd ed. A volume in Woodhead publishing series in composites science and engineering;2018.

[2] Kallannavar V, Kumaran B, Kattimani SC. Effect of temperature and moisture on free vibration characteristics of skew laminated hybrid composite and sandwich plates. Thin Wall Struct. 2020;157:107111.

[3] Wang YQ, Zu JW. Nonlinear dynamics of a translational FGM plate with strong mode interaction. Int J Struct Stab Dyn. 2018;18, 1850031.

[4] Wang YQ, Zu JW. Nonlinear dynamic thermoelastic response of rectangular FGM plates with longitudinal velocity. Compos B Eng. 2017;117:74-88.

[5] Sheng GG, Wang X. The non-linear vibrations of rotating functionally graded cylindrical shells. Nonlinear Dynam. 2017;87:1095-1109.

[6] Liew KM, Yang J, Wu YF. Nonlinear vibration of a coating-FGMsubstrate cylindrical panel subjected to a temperature gradient. Comput Methods Appl Mech Eng. 2006;195:1007-1026.

[7] Talha M, Singh BN. Large amplitude free flexural vibration analysis of shear deformable FGM plates using nonlinear finite element method. Finite Elem Anal Des. 2011;47:394-400.

[8] Akbari MR, Ghanbari J. Analytical deformation of thermoelastic stresses and deformation of functionally graded rotating hollow discs with radially varying thermo-mechanical properties under internal pressure. Comput Mater Contin. 2015;45(3):187-201.

[9] Kalali AT, Mond SH, Hassani B. Elasto-plastic stress analysis in rotating disks and pressure vessel made of functionally graded materials. Lat Am J solids Stru. 2016;13:819-834.

[10] Kolahi MRS, Karamooz M, Rahmani H. Elastic analysis of shrink-fitted thick FGM cylinders based on linear plane elasticity theory. Mech Adv Compos Struct. 2020;7:121-127.

[11] Habib ES, El-Hadek MA, El-Megharbel A. Stress analysis for cylinder made of FGM and subjected to thermo-mechanical loading. Metlas. 2018;9.

[12] Yadav OP, Jiwari R. A finite element approach to capture Turing patterns of autocatalytic Brusselator model. J Math Chem. 2019;57:769-789.

[13] Yadav OP, Jiwari R. Finite element analysis and approximation of Burger's-Fisher equation. Numer Methods Partial Differ Equ. 2017;33.

[14] Afsar AM, Go J. Finite element analysis of thermoelastic field in rotating FGM circular disk. Appl Math Model. 2010;34:33093320.

[15] Khorsand M, Tang Y. Design functionally graded rotating disks under thermoelastic loads:weight optimization. Int J Press
Vessel Pip. 2018;161:33-40.

[16] Go J. Mathematical approach to thermoelastic characteristics for functionally graded rotating disks. Appl Math Sci. 2019;13(11):533-545.

[17] Thawait AK, Sondhi L, Sanyal S, Bhowmick S. Stress and deformation analysis of clamped functionally graded rotating disks with variable thickness. Mech Mech Eng. 2019;23:202-211.

[18] Zheng Y, Bahaloo M, Mousanezhad D, Mahdi E, Vaziri A, Nayeb-Hashemi H. Stress analysis in functionally graded rotating disks with non uniform thickness and variable angular velocity. Int J Mech Sci. 2016;119:283-293.

[19] Nejad MZ, Rastgoo A, Hadi A. Exact elasto-plastic analysis of rotating disk made of functionally graded materials. Int J Eng Sci. 2014;85:47-57.

[20] Callioglu H, Sayer M, Demir E. Elastic - plastic stress analysis of rotating functionally graded discs. Thin Wall Struct. 2015;94:38-44.

[21] Kordkheili SAH, Livani M. Thermoelastic creep analysis of functionally graded various thickness rotating disk with temperature-dependent material properties. Int J Press Vessel Pip. 2013;111:63-74.

[22] Arnab B, Islam SMR, Khalak AA, Afsar AM. Finite difference solution to thermoelastic field in a thin circular FGM disk with a concentric hole. Procedia Eng. 2014;90:193-198.

[23] Allam MNM, Tantawy R, Zenkour AM. Thermoelastic stresses in functionally graded rotating annular disks with variable thickness. J Theor Appl Mech. 2018;56:1029-1041.

[24] Jalai MH, Shahriari B. Elastic stress analysis of rotating functionally graded annular disk of variable thickness using finite difference method. Math Probl Eng. 2018.

[25] Torabnia S, Aghajani S, Hemati M. An analytical investigation of elastic-plastic deformation of FGM hollow rotors under a high centrifugal effect. Int J Mech Mater Eng. 2019.

[26] Garg M. Stress analysis of variable thickness rotating FG disk. Int J Pure Appl Phys. 2017;13(1):158-161.

[27] Abdalla HMA, Casagrande D, Moro L. Thermo-mechanical analysis and optimization of functionally graded rotating disks. J Strain Anal Eng Des. 2020.

[28] Kursun A, Topcu M, Tetik T. Stress analysis of functionally disc under thermal and mechanical loads. Procedia Eng. 2011;10:2949-2954.

[29] Nkene ERA, Ngueyep LLM, Ndop J, Djiokeng ES, Ndjaka JMB. Displacement, strains and stresses investigation in an inhomogeneous rotating hollow cylinder made of functionally graded materials under an axisymmetric radial loading. World J Mech. 2018;8:59-72.

[30] Saadatfar M. Stress redistribution analysis of piezomagentic rotating thick-walled cylinder with temperature and moisture dependent material properties. J Appl Comput Mech. 202;6(1):90-104.

[31] Abrinia K, Naee H, Sadeghi F, Djavanroodi F. New analysis for the FGM thick cylinders under combined pressure and temperature loading. Am J Appl Sci. 2008;5(7):852-859.

[32] Rahimi GH, Nejad MZ. Exact solution for thermal stress in a rotating thick-walled cylinder of functionally graded materials. J Appl Sci. 2008;8(18):3267-3272.

[33] Evci C, Gulgec M. Functionally graded hollow cylinder under pressure and thermal loading:Effect of material parameters on stress and temperature distribution. Int J of Eng Sci. 2018;123:92-108. 
[34] Manthena VR, Lamba NK, Kedar GD. Mathematical modeling of thermoelastic state of a thick hollow cylinder with nonhomogeneous material properties. J Solid Mech. 2018;10(1):142-156.

[35] Yildirim V. Exact thermomechanical analysis of functionally graded (FG) thick-walled spheres. Mech Mech Eng. 2018;22(4):1197-1221.

[36] Farhan AM, Abd-Alla AM, Khder MA. Solution of a problem of thermal stresses in a non-homogeneous thermoelastic infinite medium of isotropic material by finite difference method. J Ocean Eng. 2019;4:252-262.

[37] Hu YD, Bao HJ, Xu HR. Thermoelastic strongly nonlinear vibration of rotating graded ring plate. J Theor Appl Mech. 2021;59:157-171.

[38] Go J. Thermoelastic characteristics of functionally graded circular disk models under the loading of contact forces. Math Probl Eng. 2021.

[39] Thakare S, Warbhe MS. Time fractional thermoelastic problem of a thick cylinder with non-homogeneous material properties. IOP conf Ser:Mater Sci Eng. 2021;1033.

[40] Saeedi S, Kholdi M, Loghman A, Ashrafi H, Arefi M. Thermoelasto-plastic analysis of thick-walled cylinder made of functionally graded materials using successive approximation method. Int J Press Vessel Pip. 2021;194:104481.

[41] Sharma D, Kaur R. Thermoelastic analysis of FGM hollow cylinder for variable parameters and temperature distributions using FEM. Nonlinear Eng. 2020;9:256-264.

[42] Sharma D, Kaur R. Finite element solution for stress and strain in FGM circular disk. AIP Conf Proc. 2019;2142:110024-1110024-5.

[43] Sharma D, Kaur R, Sharma H. Analysis of thermoelastic characteristics of disk using linear properties of material. Malaya J Mat. 2020;7(4):632-642.

[44] Sharma JN, Sharma D, Kumar S. Stress and strain analysis of rotating FGM thermoelastic circular disk by using FEM. Int J Pure Appl Math. 2012;74(3):339-352.

[45] Sharma JN, Sharma D, Kumar S. Vibration analysis of rotating FGM thermoelastic axisymmetric circular disk using FEM. Int J Comput Methods Eng Sci Mech. 2013;13:262-270. 